\title{
Application of Entropy Techniques in Analyzing Heart Rate Variabilityusing ECG Signals
}

\author{
M G Srinivasa \\ Department of Electronics \& Communication Engineering \\ Maharaja Institute of Technology, Thandavapura \\ Mysore, India \\ srinivasamg_ece@mitmysore.in
}

\author{
P S Pandian \\ Defence Research \& Development Organization (DRDO) \\ LRDE Campus, C V Raman Nagar, Bangalore-560 093 \\ Bangalore, India \\ pspandian@yahoo.com
}

\begin{abstract}
The variation of the heart rate about a mean value is the Heart Rate Variability (HRV). HRV reflects the functioning of cardiorespiratory control system. It is used as one of the diagnostic measures to detect heart disorders. In the proposed work, HRV analysis using entropy measures is carried out on healthy, Congestive Heart Failure (CHF) and Atrial Fibrillations (AF) subjects using their ECG signals. The entropy methods used in the work are Approximate entropy (ApE), Symbolic entropy (SyE) and Spectral entropy (SpE). ECG signals of 20 healthy subjects in the age group of $21-30$ years were acquired using dry electrode at a sampling rate of $500 \mathrm{~Hz}$ for $10 \mathrm{minutes}$. Signal processing algorithms for removal of baseline wandering, power line interference and motion artefacts were applied for the raw ECG signal. The ECG signals for $\mathrm{CHF}$ and AF subjects in the age group of $30-75$ years were obtained from the Physionet database. From the analysis it was found that values of $\mathrm{ApE}$ and $\mathrm{SyE}$ were highest for $\mathrm{AF}$ subjects and for SpE, the value was highest for healthy subjects. Further, values of all the three entropies were lowest for CHF subjects. In conclusion, it indicates that the entropy techniques are useful tools in diagnosing patients having heart disorders.
\end{abstract}

Keywords-Heart rate variability; ECG; Entropy; Congestive Heart Failure; Atrial Fibrillations;

$* * * * *$

\section{INTRODUCTION}

Heart rate variability (HRV), also known as RR variability or cycle length variability is a physiological phenomenon that gives beat-to-beat variation in the R-R interval. HRV is recognized as a powerful tool to determine the impact of the Autonomic nervous system (ANS) on heart rate [1]. The ANS which coordinates the involuntary control of cardiac muscles, smooth muscles and gland, is further divided into the parasympathetic nervous system (PNS) and the sympathetic nervous system (SNS).The parasympathetic system is involved with body energy conservation, thus reducing heart rate. Studies show that parasympathetic activity increases when the body is subjected to fatigue or sleepiness [2]. The sympathetic nervous system gets activated when the body is subjected to active or stressing condition. The effect of sympathetic nervoussystem causes an increase in heart rate, blood glucose levels and blood pressure [3]. HRV has been extensively used in clinical and diagnostic applications, as a measureof variability between successive heart beats. HRV is being used to detect drowsiness and fatigue in long distance travelling drivers [4].In the HRV time domain analysis, RR intervals are commonly mentioned as normal-to-normal intervals. This is because intervals betweensuccessive QRS complexes are generated only by normal sinus depolarizations [5]. The accurate and effective feature evaluation of HRV compared to the time domain is done by frequency domain parameters. Because of this reason, frequency domaintechniques are the most common HRV analysis methods used in research andcommercial applications [6]. The Power spectral density (PSD)analysis of HRV gives the information of power distribution of four main spectralcomponents derived from RR interval. These are ultra-low frequency (ULF), about $0.003 \mathrm{~Hz}$, very low frequency (VLF), $0.003 \mathrm{~Hz}$ to $0.04 \mathrm{~Hz}$, low frequency (LF), $0.04 \mathrm{~Hz}$ to $0.15 \mathrm{~Hz}$ and high frequency (HF) $0.15 \mathrm{~Hz}$ to $0.5 \mathrm{~Hz}$. The $\mathrm{LF}$ and $\mathrm{HF}$ components of the HRV power spectrum and the $\mathrm{LF} / \mathrm{HF}$ ratio can act asthe better indicator of fatigue.

This work emphasizes on the entropy measures of the HRV. Entropy is a measure of randomness or disorder. Various entropy techniques include Approximate entropy (ApE), Symbolic Entropy (SyE) and Spectral Entropy (SpE). ApE provides a measure of the degree of irregularity or randomness within a series of data. It is closely related to Kolmogorov entropy, which is a measure of the rate of generation of new information [7]. The parameters like $r$, for calculating ApE must be selected carefully as explained in [8]. SyE quantifies the complexity of physiological time series data [9]. SpE represents the spectral complexity of the time series data and describes the spread of power spectrum [10]. SpE is shown to be useful in predicting cardiac risks in [11]. 


\section{MATERIALS AND METHODS}

\section{A. Subjects}

A typical ECG waveform as shown Fig 1, describes a series of waves that are labelled as $\mathrm{P}, \mathrm{Q}, \mathrm{R}, \mathrm{S}$ and $\mathrm{T}$ waves. It contains an initial $\mathrm{P}$ wave followed by QRS complex and then a trailing $\mathrm{T}$ wave. The $\mathrm{P}$ wave reflects atrial depolarisation, $\mathrm{Q}, \mathrm{R}$, and $\mathrm{S}$ waves represent the ventricular depolarisation. The $\mathrm{T}$ wave corresponds to Ventricular repolarisation. The $\mathrm{U}$ wave is of indeterminate origin. The ECG has a frequency range is $0.05 \mathrm{~Hz}-100 \mathrm{~Hz}$. A normal sinus rhythm ECG signal is as shown in Fig 2. In the proposed work we have considered three categories of subjects a) Healthy b) CHF c) AF.

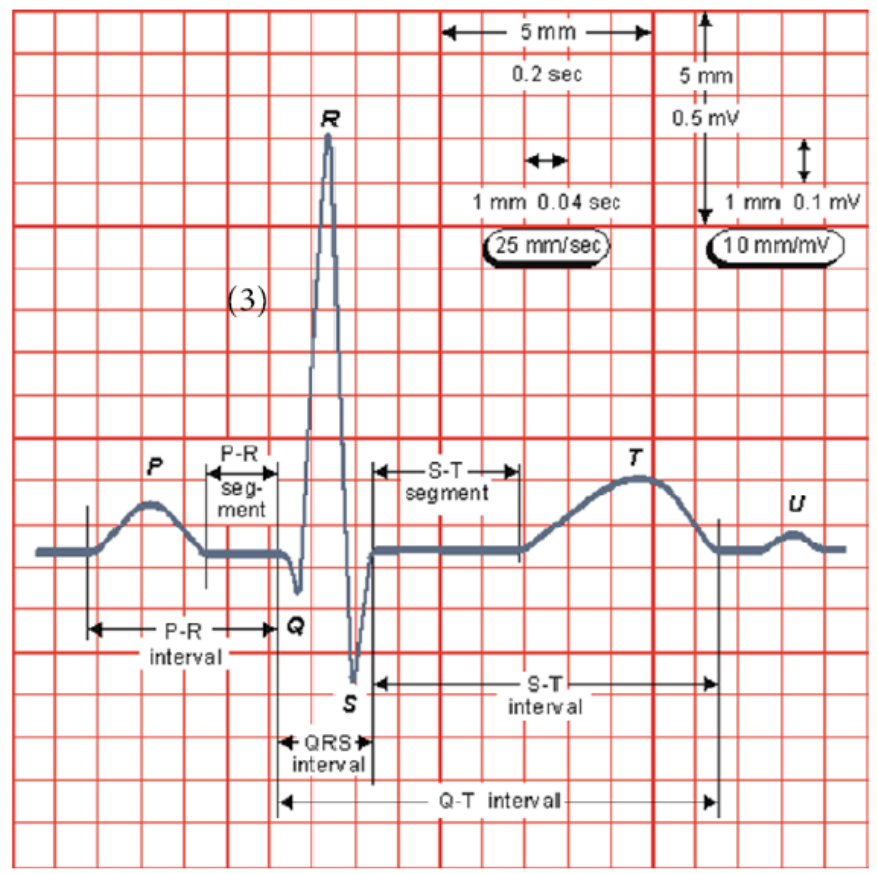

Fig 1: Typical ECG waveform

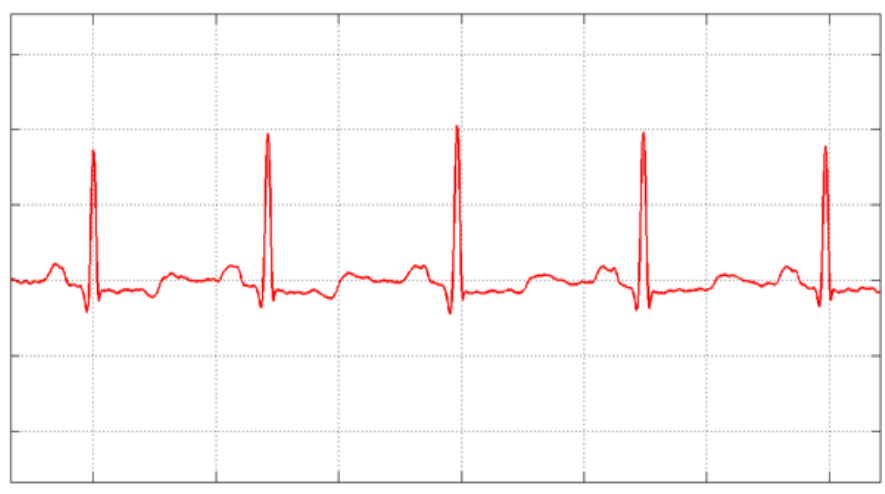

Fig 2: Normal ECG (sinus rhythm)

Healthy subjects:In this work, ECG signals of 20 healthy subjects, in the age group of 21-30 years, who do not have any cardiac diseases, were acquired in sitting position using customized dry electrode for 10 minutes at a sampling rate of $500 \mathrm{~Hz}$. Healthy subjects are characterised with higher
HRV indicating efficient autonomic mechanisms. Patients are characterised with lower HRV indicating insufficient adaptation of the ANS.

Atrial Fibrillations (AF) Subjects: AF's are a result of multiple activations, sweeping around the atrial myocardium. The AF represents absence of $\mathrm{P}$ waves. Instead of $\mathrm{P}$ waves, fibrilliform " $\mathrm{F}$ " waves with a rate higher than 350 beats per minute are present. The interval between successive QRS complexes is not constant. A typical AF waveform is as shown in Fig 3. Based on the frequency of the QRS complexes, AF can be differentiated from Bradycardia or Tachycardia. The ECG data of these subjects were acquired from the Physionet database [12].

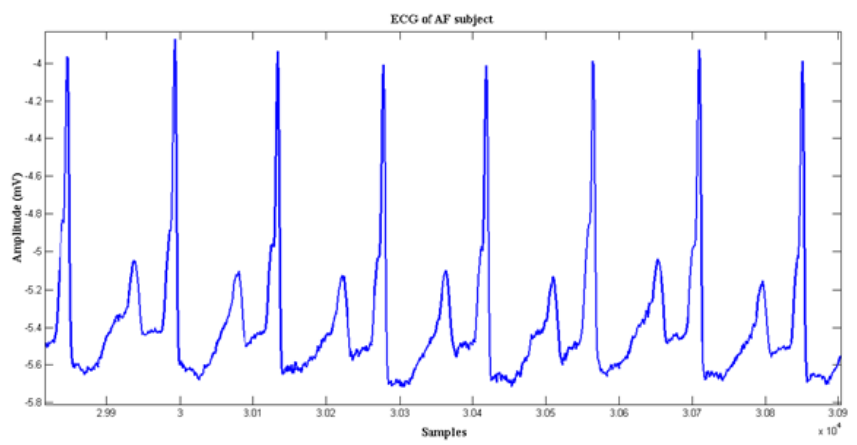

Fig 3: Atrial fibrillation

Congestive Heart Failure (CHF) Subjects:CHF is a condition in which the heart muscle is not strong enough, to pump sufficient Oxygen-rich blood to the body. Insufficient pumping of the blood from the heart leads to the accumulation of the fluid in the lungs and other organs. CHF is most common in elderly people of age above 70 years $[13,14]$. The ECG data of these subjects were acquired from the Physionet database [15]. Fig 4: CHF ECG Graph

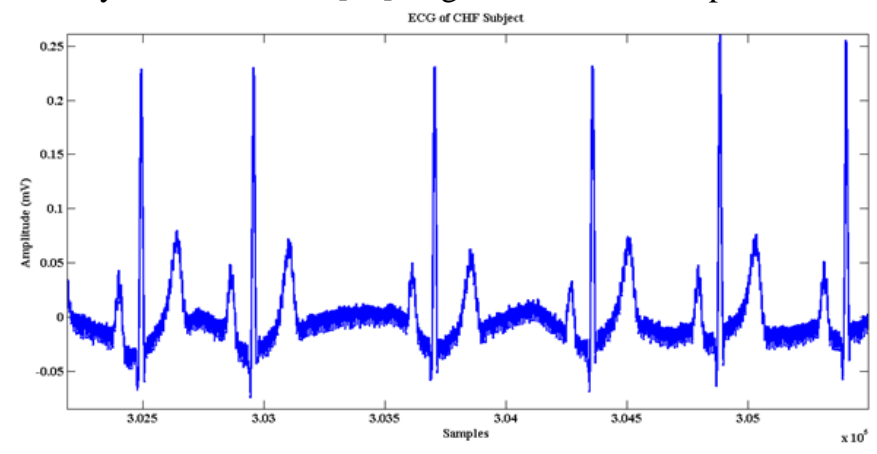

Fig 4: Congestive heart failure

\section{B. ECG signal Acquisition}

Fig 5 illustrates the block diagram used to acquire ECG signal in the Lead-I configuration. It consists of instrumentation amplifier [16] for conditioning the weak ECG signal. Further it consists of 32 bit ATMEGA microcontroller used for digitizing the raw inputs and wireless communication module for transmitting data to the remote station. The instrumentation amplifier is constructed 
using the INA128 OP-AMP which has high Slew rate and high CMRR of the order of $120 \mathrm{~dB}$. The first stage of the ECG amplifier has a gain of 10 followed by $2^{\text {nd }}$ order Butterworth Bandpass filter circuit with the frequency range of $0.5-100 \mathrm{~Hz}$. The $2^{\text {nd }}$ stage amplifier has a gain of 100 followed by a notch Filter stage used to remove the $50 \mathrm{~Hz}$ power line frequency. The power supply circuitry consists of $7.4 \mathrm{~V}, 1.2 \mathrm{AH}$ Lithium battery followed by a $5 \mathrm{~V}$ and $3.3 \mathrm{~V}$ regulator. The analog section works on $5 \mathrm{~V}$ and the digital section works on $3.3 \mathrm{~V}$. A battery charging circuit is provided for charging the battery.

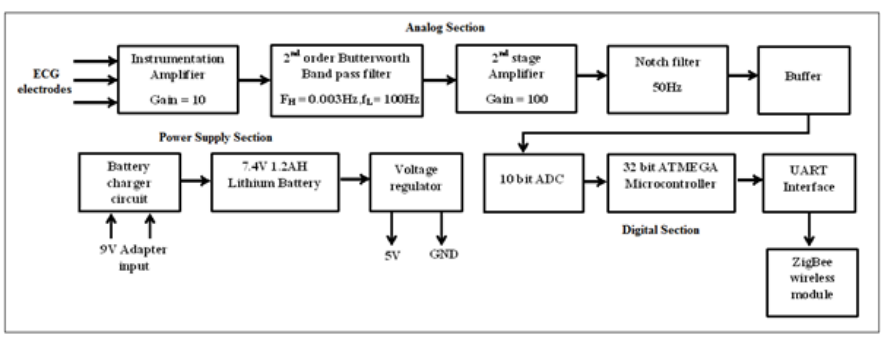

Fig 5: ECG Signal Acquisition Hardware

\section{Signal processing}

The signal acquired by the hardware consists of baseline wandering and $50 \mathrm{~Hz}$ powerline interference. These are removed from the recorded ECG data in MATLAB. Power line interference of $50 \mathrm{~Hz}$ is removed using a $50 \mathrm{~Hz}$ notch filter [17]. Baseline wandering is removed [18, 19] using wavelet decomposition technique as follows. Wavelet decomposition is performed at level 10 using db6 wavelet and the approximation and decomposition coefficients extracted. Using the approximate coefficients, the signal is reconstructed at level 8 using the same db6 wavelet. In the proposed work, entropies are calculated using the RR intervals as the input. The RR intervals are extracted from ECG signals by detecting the QRS complex from the processed signal. Fig 6 shows the block diagram for extraction of RR interval, HR and calculation of entropies. The figures show raw ECG signal and the processed signal. Fig 7: Raw ECG. Fig 8: Baseline removed ECG. Fig 9: Spectrum of raw ECG and PLI removed ECG signal.

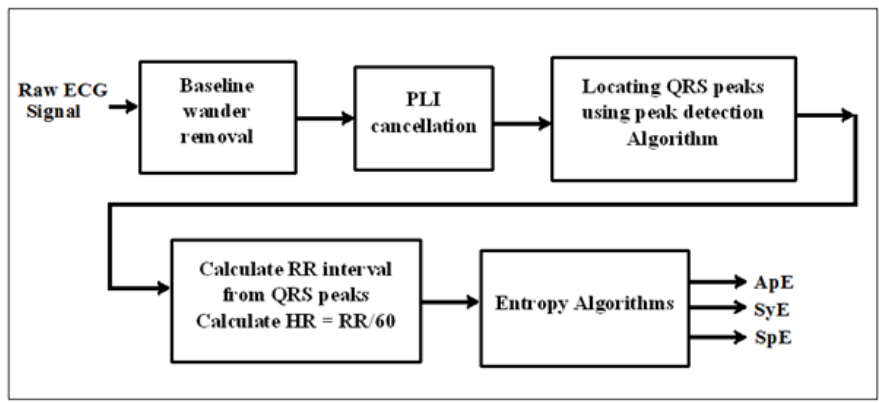

Fig 6: RR Interval and Entropy calculation algorithm

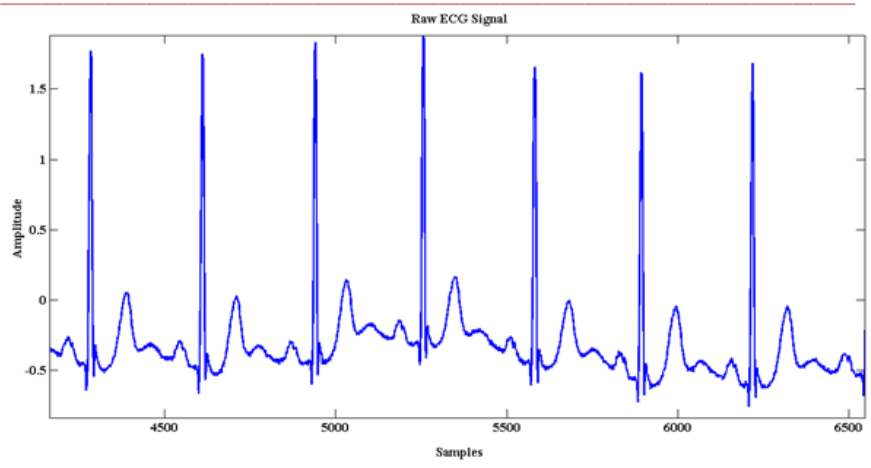

Fig 7: Raw ECG Signal

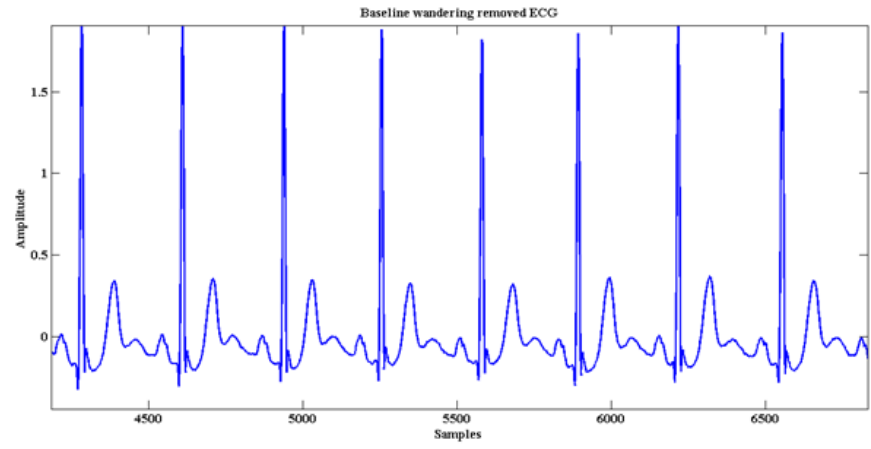

Fig 8: Baseline wander removed ECG

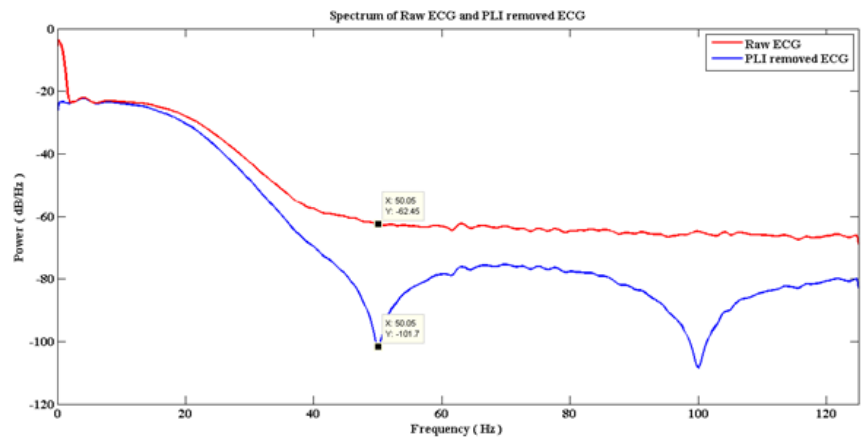

Fig 9: Spectrum of raw ECG and PLI removed ECG signal.

\section{Entropy techniques}

Approximate Entropy: It is a technique used in statistics to estimate the amount of similarity and unpredictability of fluctuations in a time-series data [20]. The algorithm to calculate ApE [21] for a time series data $u(n)$ of length $\mathrm{N}$, is as follows.

Step 1: Fix m, length of values for comparison and $r$, filtering level.

Step 2: Generate $\boldsymbol{N}-\boldsymbol{m}$ vector sequences

$$
x(1), x(2), \ldots, x(N-m+1)
$$

Where $\boldsymbol{x}(\boldsymbol{i})=[\boldsymbol{u}(\boldsymbol{i}), \boldsymbol{u}(\boldsymbol{i}+\mathbf{1}), \ldots, \boldsymbol{u}(\boldsymbol{i}+\boldsymbol{m}-\mathbf{1})]$

Step 3: For each $\boldsymbol{i}, \quad \boldsymbol{i} \leq \mathbf{1} \leq \boldsymbol{N}-\boldsymbol{m}+\mathbf{1}$, Calculate $C_{i}^{r}(r)=$ number of $x(j)$ such that $d[x(i), x(j)] \leq r$ 
Where $d\left[x, x^{*}\right]=\max \left|\boldsymbol{u}(\boldsymbol{a})-\boldsymbol{u}^{*}(\boldsymbol{a})\right|$

Step 4: Calculate

$$
\boldsymbol{\varphi}^{\boldsymbol{m}}(\boldsymbol{r})=(\boldsymbol{N}-\boldsymbol{m}+\mathbf{1})^{-\mathbf{1}} \sum_{i=1}^{N-m+1}\left[\log \boldsymbol{C}_{i}^{\boldsymbol{r}}(\boldsymbol{r})\right]
$$

Step 5: Approximate Entropy,

$$
A p E=\varphi^{m}(r)-\varphi^{m+1}(r)
$$

In this work, $\mathrm{m}=2$ and $\mathrm{r}=0.2$ are selected [22].

Symbolic Entropy: SyE quantifies the complexity of physiological signal. The concept here is to convert the time domain signal into a sequence of symbols based on a predefined threshold. The Shannon entropy of these symbols itself is the SyE [9]. SyE for time domain signal $\boldsymbol{u}(\boldsymbol{n})$ is calculated as follows. Let

$$
u(n)=[72,73,78,81,83,84,89,92,94]
$$

Step 1: Calculate the difference between each sample and its next sample.

$$
\begin{aligned}
& d(n)=[u(1)-u(0), u(2)-u(1), u(3) \\
& -u(2), \ldots, u(N)-u(N-1)] \\
& d(n)=[1,5,3,2,1,5,3,2]
\end{aligned}
$$

Step 2: Form sequence $\boldsymbol{S}(\boldsymbol{n})$ such that,

$$
S(n)= \begin{cases}1, & \text { if } d(n) \geq T \\ 0, & \text { if } d(n) \leq T\end{cases}
$$

Here threshold $\mathrm{T}$ is set to mean of the signal [23], $\boldsymbol{d}(\boldsymbol{n})$. Here $\boldsymbol{T}=\mathbf{2 . 7 5}$

$$
\text { Then } S(\boldsymbol{n})=[\mathbf{0}, \mathbf{1}, \mathbf{1}, \mathbf{0}, \mathbf{0}, \mathbf{1}, \mathbf{1}, \mathbf{0}]
$$

Step 3: Convert $\boldsymbol{S}(\boldsymbol{n})$, into sequence of symbols, $\boldsymbol{K}(\boldsymbol{n})$, using 4 bits each.

$$
\begin{gathered}
K(n)=[0110,1100,1001,0011,0110] \\
K(n)=[6,12,9,3,6]
\end{gathered}
$$

Step 4: Symbolic entropy is the Shannon entropy for this sequence $\boldsymbol{P}(\boldsymbol{n})$, calculated using

$$
S y E=-\sum P(i) \log P(i)
$$

Where $\boldsymbol{P}(\boldsymbol{n})$ is the probability of occurrence of symbols $\operatorname{in} K(n) . P(n)=[0.4,0.2,0.2,0.2,0.4]$

$$
S y E=1.6986
$$

Spectral Entropy: The spectral entropy of a signal is a measure of its spectral power distribution [24]. The concept is based on the Shannon entropy in information theory. The $\mathrm{SpE}$ treats the signal's normalized power distribution in the frequency domain as a probability distribution [25, 26], and calculates the Shannon entropy of it. The spectral entropy is calculated by the following steps.

Step 1: Computation of Discrete Fourier Transform of the time domain HRV data, $\boldsymbol{x}(\boldsymbol{n})$

$$
X(K)=\sum_{n=0}^{N-1} x(n) W_{N}^{k n} \quad, 0 \leq K \leq N-1
$$

Where $W_{N}=e^{\frac{-j 2 \pi}{N}}$

Step 2: Computation of power spectral density of the HRV signal using the formula

$$
S(k)=\frac{1}{N}|X(K)|^{2}
$$

Step 3: Calculation of normal probability density function using the formula

$$
P_{k}=\frac{S(k)}{\sum_{i} S(i)}
$$

Step 4: The spectral entropy is calculated using the Shannon entropy using the formula

$$
S p E=-\sum P_{k} \log P_{k}
$$

\section{RESULTS}

Fig. 7 shows the raw ECG signal acquired from healthy subject. The ECG signal after removing the baseline wandering is shown in Fig. 8. Spectrum of the raw ECG signal before filtering and the spectrum of the $50 \mathrm{~Hz}$ PLI removed signal is shown in Fig 9. From the graph it shows that power at $50 \mathrm{~Hz}$ before filtering is $-62.45 \mathrm{~dB} / \mathrm{Hz}$ and after filtering, the power is $-101.7 \mathrm{~dB} / \mathrm{Hz}$.

Fig 10 shows the ApE values of the 20 healthy subjects. The result indicates that the average ApE value for healthy subject is 1.539. Fig 11 shows the comparison of ApE values of healthy, $\mathrm{CHF}$ and $\mathrm{AF}$ subjects. The graph indicates that the AF subjects have higher ApE and CHF subjects have lower ApE values for 5 subjects. Table 1 shows the average ApE values. Fig 12 shows the average ApE for values for different types of subjects. 


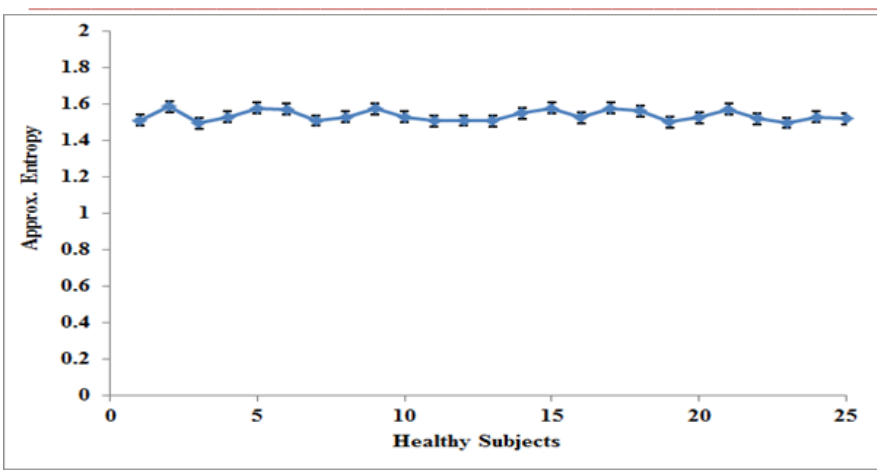

Fig 10: Approximate entropy of 20 healthy subjects

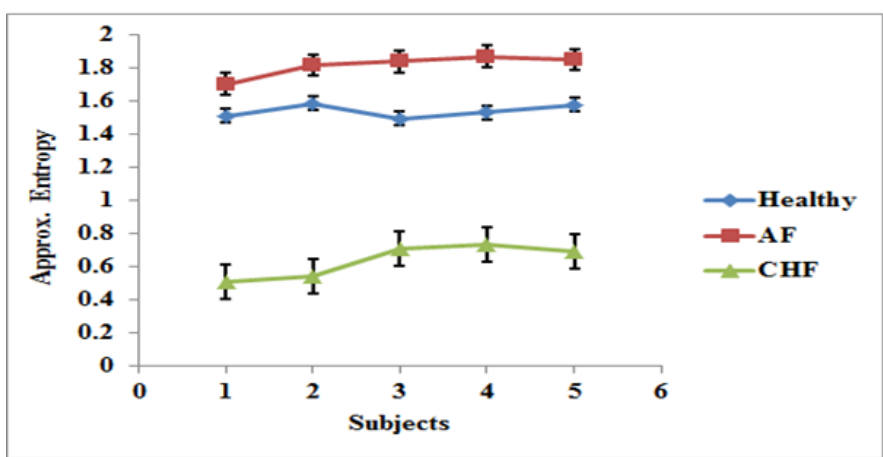

Fig 11: Variation of approximate entropy values for five healthy, AF and $\mathrm{CHF}$ subjects

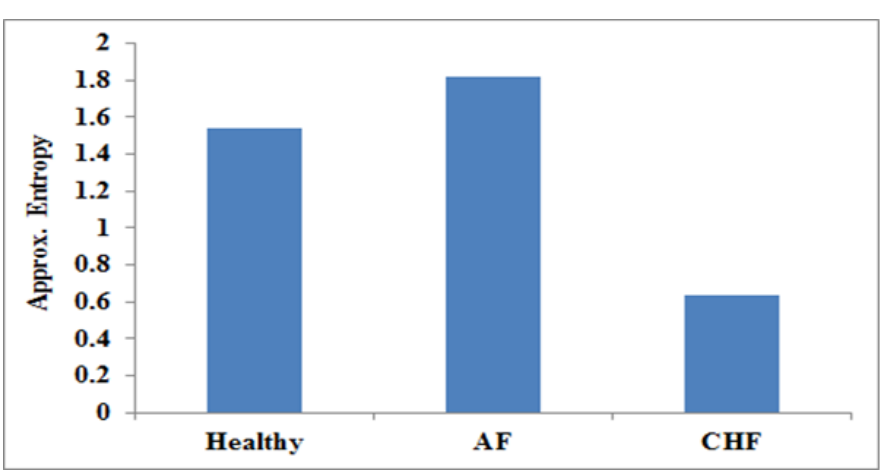

Fig 12: Comparison of approximate entropy for healthy, AF and $\mathrm{CHF}$ subjects

Fig 13 shows SyE values of the 20 healthy subjects.The result indicates that the average $\mathrm{SyE}$ value for healthy subject is 1.24 . Fig 14 shows the comparison of SyE values of healthy, CHF and AF subjects. The graph indicates that the AF subjects have higher SyE and CHF subjects have lower SyE values for 5 subjects. Table 2 shows the average SyE values. Fig.15 shows the average SyE for values for different types of subjects.

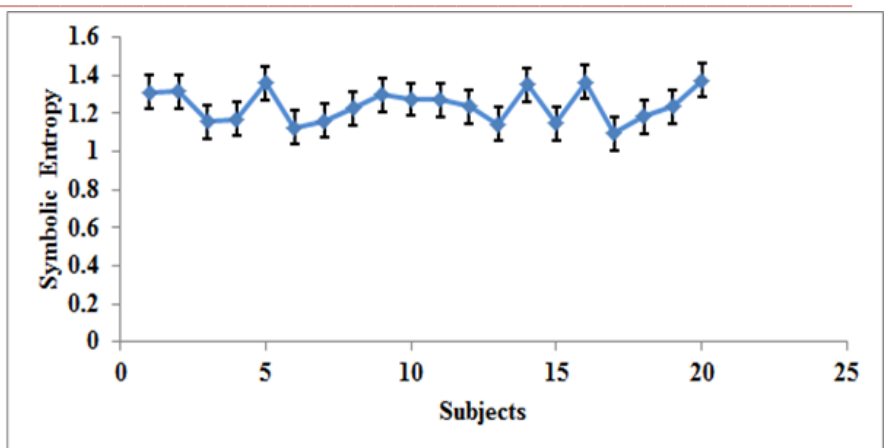

Fig 13: Symbolic entropy of 20 healthy subjects

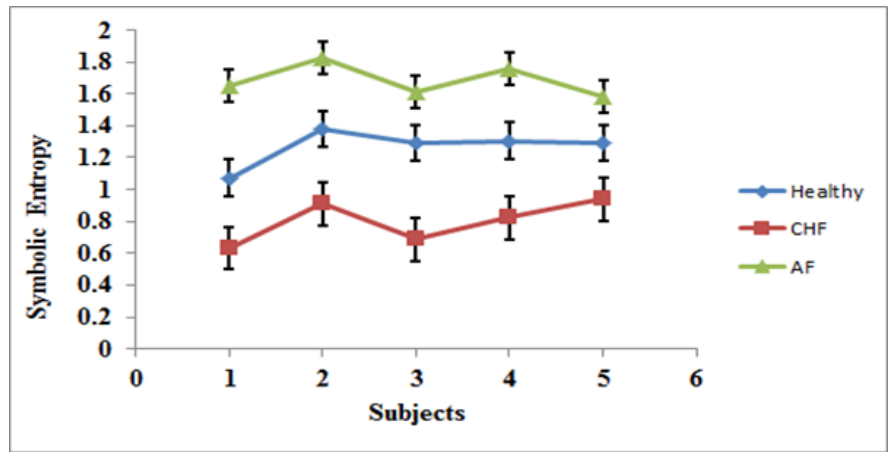

Fig 14: Variation of symbolic entropy values for five healthy, AF and CHF subjects

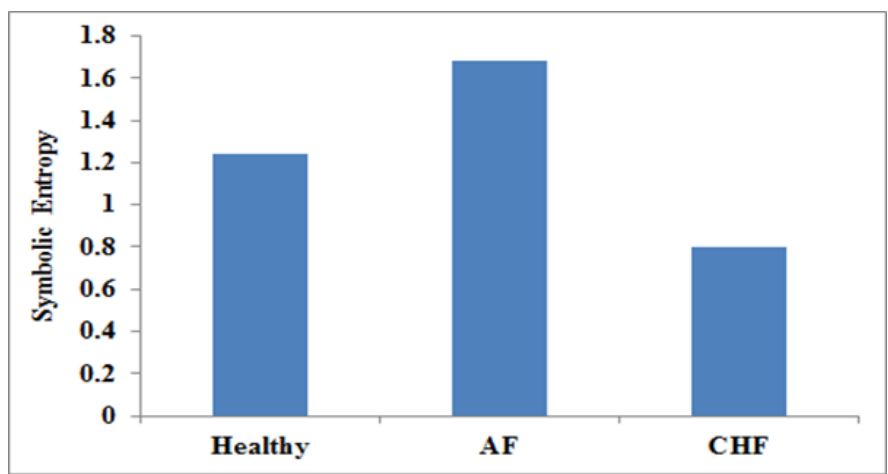

Fig 15: Comparison of symbolic entropy for healthy, AF and CHF subjects

Fig 16 shows $\mathrm{SpE}$ values of the 20 healthy subjects. The result indicates that the average $\mathrm{SpE}$ value for healthy subject is 2.28. Fig 17 shows the comparison of $\mathrm{SpE}$ values of healthy, CHF and AF subjects. The graph indicates that the healthy subjects have higher SpE and CHF subjects have lower SpE values for 5 subjects.[10,27] Table 3 shows the average $\mathrm{SpE}$ values. Fig 18 shows the average $\mathrm{SpE}$ for values for different types of subjects.

Fig 19 shows a HRV power spectral density of healthy subjects. From the graph it is seen that power density is distributed from $0-0.2 \mathrm{~Hz}$ including both low and high frequency ranges. Fig 20 shows a HRV power spectral density of CHF subjects. From the graph it is seen that power density is concentrated only in the low frequency range of $0-0.15 \mathrm{~Hz}$. Fig 21 shows a HRV power spectral 
density of AF subjects. From the graph it is seen that power density is widely distributed among all the frequency ranges from $0-0.5 \mathrm{~Hz}$.

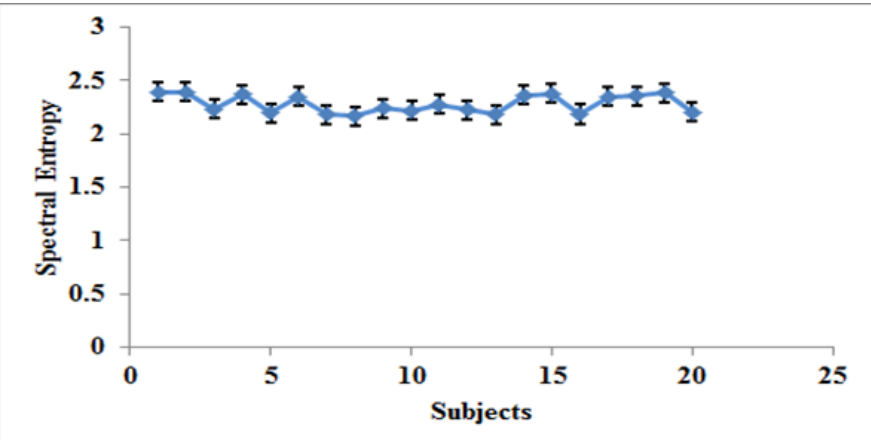

Fig 16: Spectral entropy of 20 healthy subjects

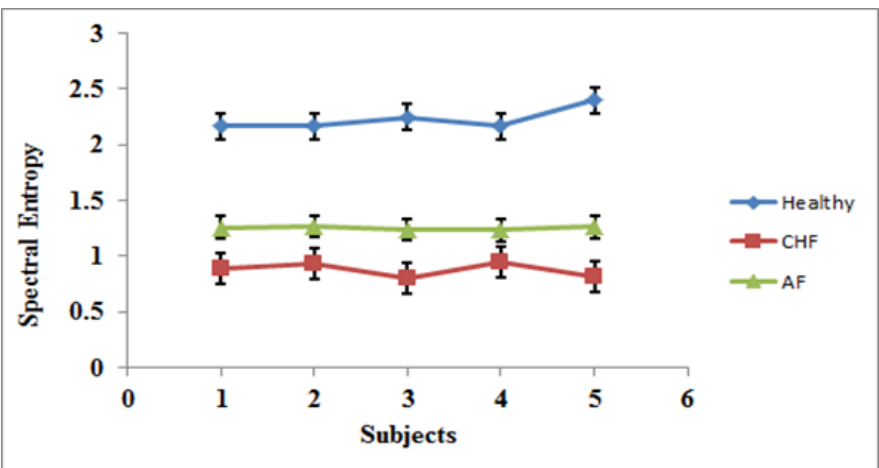

Fig 17: Variation of spectral entropy values for five healthy,

$\mathrm{AF}$ and $\mathrm{CHF}$ subjects

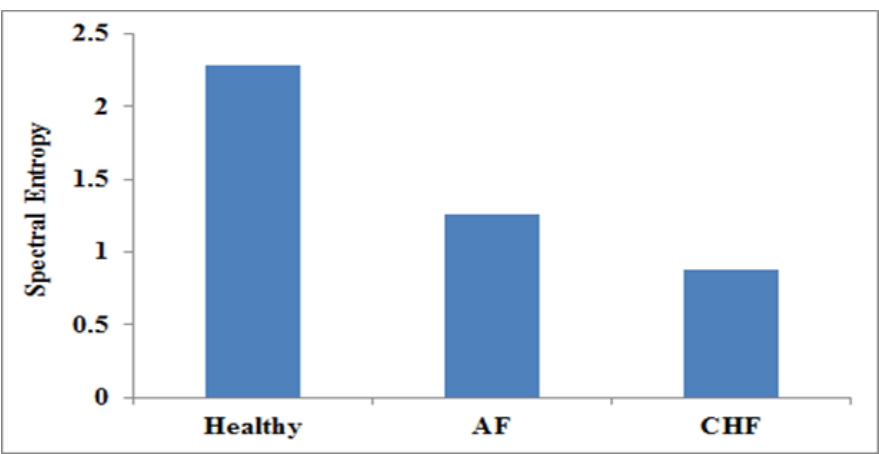

Fig 18: Comparison of spectral entropy for healthy, AF and

CHF subjects

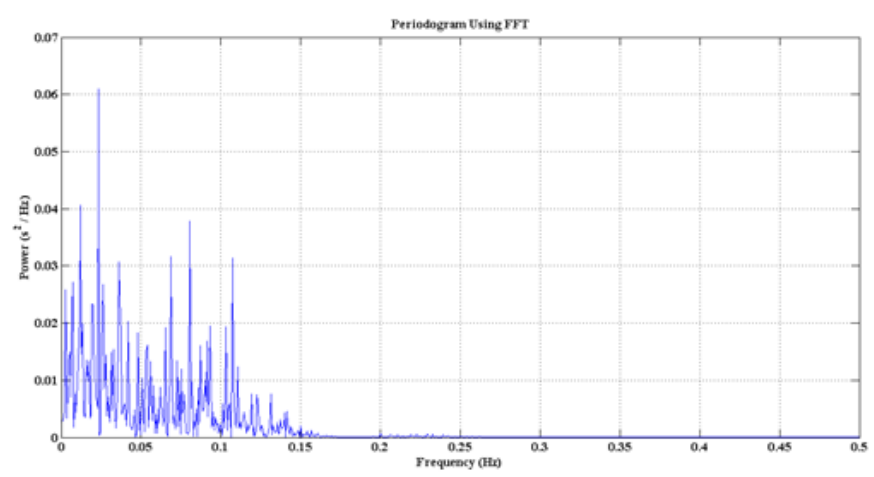

Fig19: Power Spectral Density of healthy subject

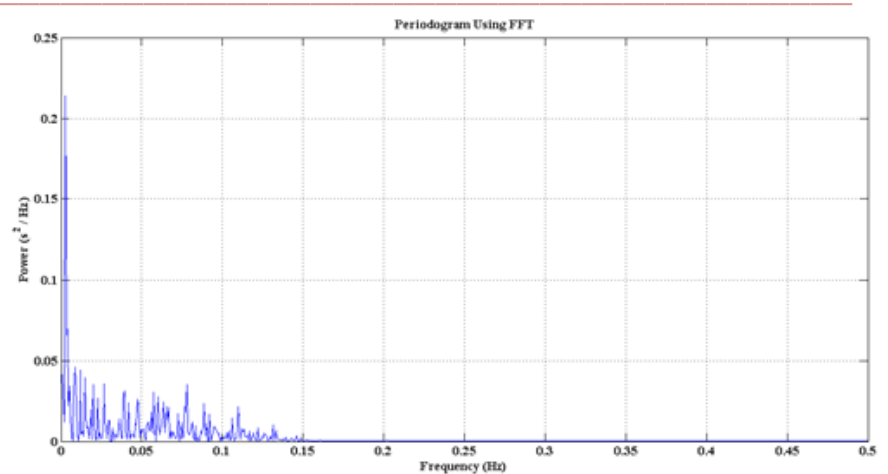

Fig20: Power Spectral Density of CHF subject

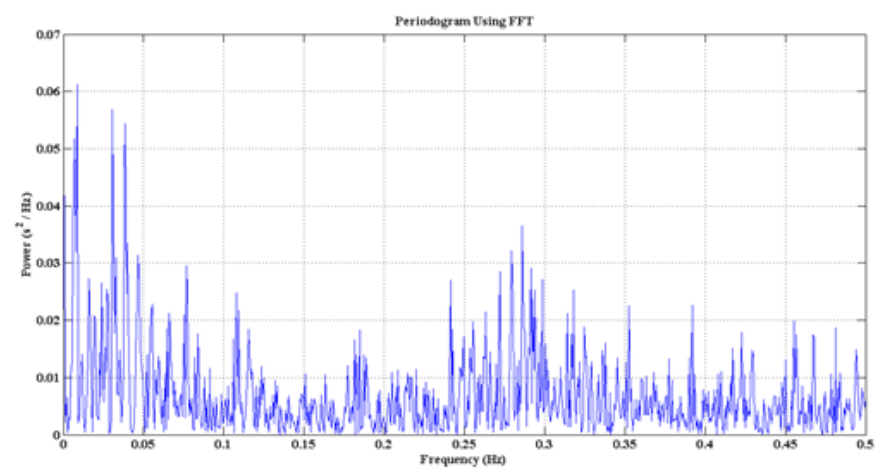

Fig21: Power Spectral Density of AF subject

\section{CONCLUSION}

In the proposed work, the HRV analysis is carried out using Entropy techniques. It can be seen that entropy techniques are a good measure of differentiating $\mathrm{CHF}$ and $\mathrm{AF}$ from healthy subjects. Atrial Fibrillations are caused by multiple activations around the atrial myocardium. As a result there is more irregularity in the heart rate of such subjects. These irregularities can be analysed using the entropy techniques. Thus from the tables 1 and 2, it is seen that, for AF subjects the entropy value is highest as compared to healthy and CHF subjects. Tables 1, 2 and 3, suggest that entropy vales for CHF subjects are least. This is because of insufficient pumping of blood from the heart.

Table 1: The average Approximate Entropy values of Healthy, AF, CHF subjects

\begin{tabular}{|c|c|}
\hline Subject Type & Approximate Entropy \\
\hline Healthy & $1.53992 \pm 0.04$ \\
\hline AF & $1.8161 \pm 0.065$ \\
\hline CHF & $0.63792 \pm 0.103$ \\
\hline
\end{tabular}


Table 2: The average Symbolic Entropy values of Healthy, $\mathrm{AF}, \mathrm{CHF}$ subjects

\begin{tabular}{|c|c|}
\hline Subject Type & Symbolic Entropy \\
\hline Healthy & $1.24024 \pm 0.088$ \\
\hline AF & $1.68506 \pm 0.135$ \\
\hline CHF & $0.79885 \pm 0.101$ \\
\hline
\end{tabular}

Table 3: The average Spectral Entropy values of Healthy,

$\mathrm{AF}, \mathrm{CHF}$ subjects

\begin{tabular}{|c|c|}
\hline Subject Type & Spectral Entropy \\
\hline Healthy & $2.282005 \pm 0.086$ \\
\hline AF & $1.25402 \pm 0.068$ \\
\hline CHF & $0.87894 \pm 0.013$ \\
\hline
\end{tabular}

From table 4, it is seen that approximate entropy is a good method to differentiate CHF subjects from healthy subjects and symbolic entropy is good method to differentiate $\mathrm{CHF}$ subjects from AF subjects. From spectral analysis, it is observed that the HRV distribution is concentrated in the low frequency ranges $0-0.15 \mathrm{~Hz}$ for healthy subjects but it is widely distributed over a larger frequency range up to $0.5 \mathrm{~Hz}$ for AF subjects. Hence the spectral entropy is higher for healthy subjects than that of AF or CHF subjects. This is as shown in Tables 3 and 4. It is seen that spectral entropy is a good method to differentiate healthy and diseased subjects.

Table 4: Comparison of different entropies.

\begin{tabular}{|c|c|c|c|c|}
\hline $\begin{array}{c}\text { Subject } \\
\text { type }\end{array}$ & $\begin{array}{c}\text { Approx. } \\
\text { Entropy } \\
\text { (ApE) }\end{array}$ & $\begin{array}{c}\text { Symbolic } \\
\text { Entropy } \\
\text { (SyE) }\end{array}$ & $\begin{array}{c}\text { Average } \\
\text { of ApE } \\
\text { \& SyE }\end{array}$ & $\begin{array}{c}\text { Spectral } \\
\text { Entropy } \\
\text { (SpE) }\end{array}$ \\
\hline Healthy & 1 & 1 & 1 & 1 \\
\hline $\mathrm{AF}$ & 1.17 & 1.35 & 1.26 & 0.55 \\
\hline $\mathrm{CHF}$ & 0.41 & 0.64 & 0.52 & 0.39 \\
\hline
\end{tabular}

From the above work, we can conclude the following points in the implementation of the three entropy techniques. The approximate entropy algorithm is the simplest technique for calculating the entropy. But when the data length, N, changes the entropy value will also change. The symbolic entropy algorithm is good for shorter data lengths. But when the data length is increased, the symbolic bit size for comparison must be increased. Otherwise the algorithm takes more time to compute the entropy values. The spectral entropy algorithm works on frequency domain data. Hence the time series data is resampled and converted to frequency domain using FFT techniques. Here we have to use windowing techniques for better results [28]. Hence this algorithm is more complex compared to the above two algorithms.

\section{ACKNOWLEDGMENT}

The authors would like to thank Dr. Karthik Udupa of Vatsalya Hospital Mysore, India for helping us in cross verifying the healthy subject's data collected by us and gave good suggestion in separating the data of $\mathrm{CHF} \& \mathrm{AF}$ subjects. This study was supported by Maharaja Education Trust, Mysore, India.

\section{REFERENCE}

[1] Oweis, R. J. and Al-tabbaa, B. O. (2014), 'QRS Detection and Heart Rate Variability Analysis : A Survey QRS Detection and Heart Rate Variability Analysis : A Survey', Biomedical Science and Engineering 2(January), 13-34.

[2] Jung, S.-J., Shin, H.-S. and Chung, W.-Y. (2014), 'Driver fatigue and drowsiness monitoring system with embedded electrocardiogram sensor on steering wheel', IET Intelligent Transport Systems 8(1), 43-50.

[3] Uemura, E. (2015), Autonomic Nervous System

[4] E.Billman, G., V.Huikuri, H., Sacha, J. and Trimmel, K. (2015), 'An introduction to heart rate variability : methodological considerations and clinical applications', Frontiers in Physiology 6(February), 1-3.

[5] Milagro, F. J. (2016), Poincare Plot Analysis and Graphical User interface Development for the Study of Heart, PhD thesis.

[6] E.Billman, G., V.Huikuri, H., Sacha, J. and Trimmel, K. (2015), 'An introduction to heart rate variability: methodological considerations and clinical applications',Frontiers in Physiology 6(February), 1-3.

[7] CH. RenuMadhavi and A.G. Ananth, "Analysis and Characterisation of Heart Rate Variability (HRV) Data of Different Sets of Subjects Using Nonlinear Measure (Approximate Entropy)", International Journal of Computer Theory and Engineering, Vol 2(4), pp 619-623, 2010.

[8] Amritpal Singh, and Jashandeep Kaur, Approximate Entropy (ApEn) based Heart Rate Variability Analysis, Indian Journal of Science and Technology, Vol 9(47), December 2016.

[9] CH. RenuMadhavi and A.G. Ananth, "Quantification of Heart Rate Variability(HRV) Data using Symbolic Entropy to distinguish between healthy and disease subjects.", International Journal of computer Applications( IJCA) ,Volume 8,No.12,pp10-12,2010.

[10] CH.RenuMadhavi and A.G. Ananth, "Quantification of Healthy, Cardiac and Non-cardiac diseased subjects using Spectral Entropy", International Journal of 
Biomedical Engineering and Consumer Health Informatics, July-Dec 2012.

[11] CH.RenuMadhavi,Estimation of Spectral Entropy of HRV Data and its Application to Depression and Thyroid Subjects to Predict Cardiac Risk,Biomedical \& Pharmacology Journal, September 2018.

[12] https://physionet.org/physiobank/database/afdb/ , Atrial Fibrillation Database

[13] Chua Kuang Chua, Vinod Chandran, Rajendra U Acharya and Lim Choo Min, "Cardiac health diagnosis using higher order spectra and support vector machine", Open Med Information J,3:pp1-8, 2009.

[14] Acharya UR, Kannathal N, and Krishnan SM, "Comprehensive analysis of cardiac health using heart rate signals", PhysiolMeasJ, 25:pp1130-1151, 2004a

[15] https://physionet.org/physiobank/database/chfdb/ ,Congestive Heart Failure Database.

[16] Murugavel Raju, Heart-Rate and EKG Monitor Using the MSP430FG439, Application Report, SLAA280A-October 2005-Revised September 2007

[17] Ms. Geeta Kadam, Prof.P.C.Bhaskar, REDUCTION OF POWER LINE INTERFERENCE IN ECG SIGNAL USING FIR FILTER, IJCER | Mar-Apr 2012 | Vol. 2 | Issue No.2 |314-319

[18] Na Pan, VaiMang, Mai Peng Un, Pun Sio hang, Accurate Removal of Baseline Wander in ECG Using Empirical Mode Decomposition, Proceedings of NFSI \& ICFBI 2007 Hangzhou, China, October 12-14, 2007

[19] A. Kumar et al.A Novel Approach in ECG Signal Processing, Signal and Image Processing in Medical Applications, SpringerBriefs in Forensic and Medical Bioinformatics, DOI 10.1007/978-981-10-0690-6_2

[20] Roberto Hornero, Aboy, Member, Daniel Abasolo, James McName and BrahmGoldstein, "Interpretation of Approximate Entrophy: Analysis of Intra Cranial Pressure Approximate EntrophyDuring Acute Intracranial Hyper tension" IEEE Transactions on Bio-Medical Engineering, 52(10), pp 1671-1680, 2005.

[21] Yang Fusheng, Hongbo and Tang Quingyu, "Approximate entropy and it's application in bio signal analysis", chapter 3, pp72-99

[22] CH.Renumadhavi and A.G.Ananth "Multiple scale Sample entropy of Heart Rate Variability (HRV)of normal and congestive heart failure cases by selecting ' $\mathrm{m}$ ' and ' $\mathrm{r}$ ' in " Journal of Material Science and Engineering, Volume1(34), No.9, pp66-71,2010

[23] K.T. Park and S.H. Yi, "Accessing physiological complexity of HRV using threshold dependent symbolic entropy", Journal of Korean Physical Society, Vol 44, No.3, pp569-576, 2004.

[24] Aihuazhang, Bin Yang, and Ling huang, "Feature extraction of EEG signal using power spectral entropy", Proceedings of international conference on biomedical engineering and informatics, vol(2),pp435-439, 2009.

[25] Mehmet Engin,SerkanUnsal,ErkanZekiEngin,, “Order selection in Autoregressive power spectrum estimation of sleep EEG” ELECO-2001,Second International Conference on Electrical and

[26] Electronics Engineering,7-11,November2001,Bursa,Turkey
[27] Aihuvazhang, Bin Yang,Ling Huang, "Feature extraction of EEG using power spectral entropy", International conference on biomedical engineering and informatics2008.

[28] V. C.Veera Reddy and Anuradha, "Cardiac arrhythmia classification using fuzzy classifiers, Journal of theoretical and applied information technology", pp352-359,2005.

[29] B.S.Saini, Dilbag Singh, Moin Uddin and Vinod Kumar "Improved power spectrum estimation for RR interval time series" World Academy of Science and Technology, 46pp44-48, 2008. 